\title{
Environmental control of defensive reactions to footshock
}

\author{
ROBERT J. BLANCHARD, KENNETH K. FUKUNAGA, and D. CAROLINE BLANCHARD \\ University of Hawaü, Honolulu, Hawaï 96822
}

Footshock given immediately after rats are placed in a strange inescapable environment produces no diminution in movement. However, if a brief exploration period is allowed before shock, freezing is the dominant defensive behavior seen. Thus, the topographical similarity of postshock reactions to unconditioned defensive behaviors elicited by a cat also involves a similar flight-to-freezing shift when the subject is permitted prior familiarization with the inescapable threat situation.

Blanchard, Fukunaga, and Blanchard (1976) have reported that a brief familiarization period in a novel, inescapable chamber changes the dominant defensive behavior of a rat to a cat in that chamber, from flight to freezing. In view of the similarity between cat-elicited defensive behaviors and reactions to conditioned threat stimuli (Blanchard \& Blanchard, 1971), it seems likely that a similar shift might occur with familiarization of a chamber in which footshock is given. Thus, in order to assess the generality of effects of familiarity with the inescapable test situation on defensive reactions to threat, the procedure employed in Blanchard, Fukunaga, and Blanchard (1976) was repeated, using shock rather than a cat as the threat stimulus.

\section{METHOD}

\section{Subjects}

Twenty-four naive albino rats from 135 to 192 days of age were randomly assigned to three groups of eight rats each. These rats were from the colony maintained by the University of Hawaii Psychology Department.

\section{Apparatus}

A flat-gray $50 \times 50 \times 15 \mathrm{~cm}$ wooden box with a solid wood cover containing an $18 \times 18 \mathrm{~cm}$ Plexiglas insert served as the test chamber. The chamber was placed on a grid floor consisting of $64-\mathrm{cm}$ stainless steel rods which were suspended over a Formica base divided by lines into four $25 \times 25 \mathrm{~cm}$ areas.

Shock was delivered to the grids by a Grason-Stadler E1064 shock source. A $60-\mathrm{W}$ bulb suspended $20 \mathrm{~cm}$ over the Plexiglas insert in the ceiling of the chamber provided illumination. A 65-db SPL speech noise from a Grason-Stadler noise generator was provided throughout the experiment.

\section{Procedure}

The three groups of the present experiment were identical to those of Blanchard, Fukunaga, and Blanchard (1976), except that shock instead of a cat was used as the threatening stimulus. The no-shock group was left in the apparatus for a 7-min period; the immediate-shock group was given a single 3-mA shock, 2 sec in duration, immediately upon placement in the test chamber; the delayed-shock group was left in the apparatus for

Requests for reprints should be sent to Robert J. Blanchard, University of Hawaii, Department of Psychology, 2430 Campus Road, Honolulu, Hawaii 96822.
$2 \mathrm{~min}$, then briefly lifted and replaced at the same time that a shock of identical intensity and duration as that of the immediate-shock group was given. Both shock groups were observed for $5 \mathrm{~min}$ after shock, and measurements of movement times were made each minute that a rat was in the apparatus. Since shock always elicited jumping and running, and these movements persisted for brief periods after the shock terminated, ratings of initial defensive reactions to the shocks were not taken.

\section{RESULTS AND DISCUSSION}

Movement times for each minute of the test sessions are presented in Figure 1. The delayed-shock and noshock control groups did not differ $[\mathrm{t}(14)=.94$, $p>.05]$ in their activity during the first $2 \mathrm{~min}$ in the test apparatus. After shock was given, however, the delayed-shock group showed a marked decrease in movement times, accompanied by freezing $[t(14)=27.97$, $\mathrm{p}<.001$, for movement times of no-shock and delayed-

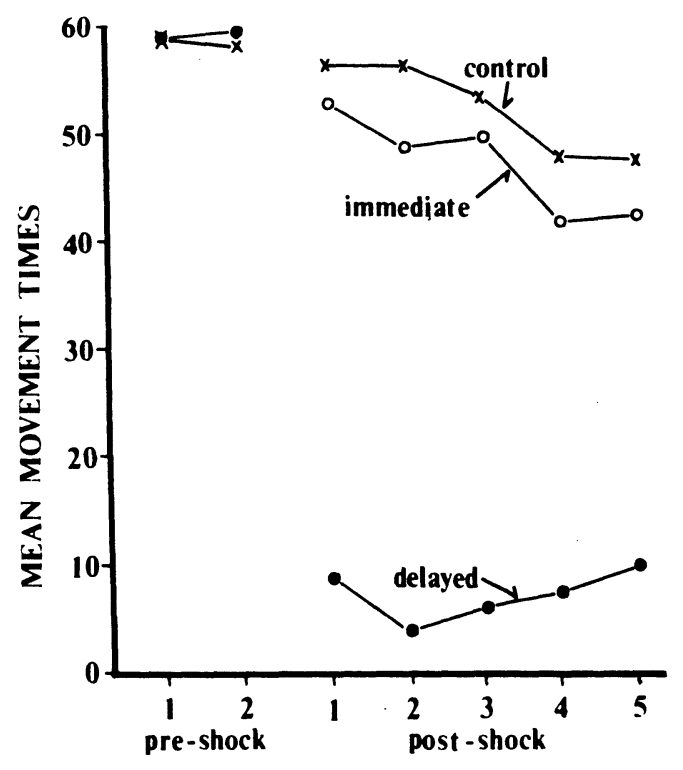

ONE-MINUTE PERIODS

Figure 1. Mean movement times for each $2 \mathrm{~min}$ of the test periods, for rats given shock immediately after placement in the test situation, or after a delay, and for nonshocked controls. 
shock groups during 2 min after shock for the delayedshock group]. The immediate-shock group showed an intermediate level of suppression during this same period, reliably less $[\mathrm{t}(14)=11.43, \mathrm{p}<.001]$ than the delayed-shock group, but also reliably greater (i.e., less movement time) than for the no-shock group [ $t(14)=$ $2.17, \mathrm{p}<.05]$. However, unlike the movement time differences of delayed and immediate groups confronted by a cat (Blanchard et al., 1976), the present delayedshock and immediate-shock groups' movement time differences did not disappear with increasing time (up to $5 \mathrm{~min}$ ) after shock: During the last $3 \mathrm{~min}$ after shock, movement time scores for these groups.were still divergent, with less movement (greater suppression) for the delayed-shock group $[t(14)=3.89, p<.01]$. Other than this last finding (which may possibly reflect traumalinked impairment of the perceptual or cognitive activities or abilities of the subjects), suppression of movement to footshock closely resembles the suppression seen after confrontation with a cat: Delay in presentation of the threat stimulus enhances freezing, apparently by reducing or eliminating the abortive attempts at flight or escape which predominate when the rat is confronted by threat in a totally new situation. Thus, the present experiment extends the generality of findings (Blanchard et al., 1976) that the specific topology of defensive behavior may be determined by familiarity with important aspects of the situation in which threat is encountered.

This interpretation obviously relies on an assumption that the 2-min exposure to the novel and inescapable situation resulted in the subjects' discovery that the situation was, in fact, inescapable. There is, moreover, direct evidence on this point: When placed in a novel situation of very similar dimensions to those of the present test chamber, naive rats confronted with a cat found and exited through a doorway with an average first-trial latency of about $30 \mathrm{sec}$. On the second trial in the same situation, latencies for the experimental (cat) group showed a reliable reduction to a mean of approximately $5 \mathrm{sec}$, clearly indicating that the animals had learned an exit was available. Finally, control rats (no cat) had first-trial exit latencies similar to those of the experimental group, indicating that much less than 2 min was needed to find and use an exit from such a novel situation, even when there was no extraneous motivation to do so (Blanchard \& Blanchard, 1971).
In summary, the results of the present experiment, like those of Blanchard et al. (1976), clearly indicate that punishment effects do not mediate the determination of defensive response topology by features of the threat situation. The indication is that these features may directly elicit differential defensive behaviors.

In addition to the theoretical interest in this finding, it is also relevant to a problem of considerable importance in the avoidance conditioning literature: Since flight and freezing are mutually incompatible, the joint elicitation of both reactions may produce a very variable behavior pattern in which it is difficult to assess the magnitude of either response tendency. Since many psychologists study defensive responses only in order to evaluate their effects on experimenter-chosen avoidance behaviors such as barpressing or jumping over a barrier, it is of major importance for these investigators to be able to predict and control defensive responses in the specific tasks they use.

In this connection, the present results suggest that such factors as the rat's familiarity with the presence or absence of an escape route may be a decisive factor in producing flight as opposed to freezing. Repeated punishment of attempts to flee may enhance the subject's perception of the situation as inescapable, but the present studies clearly indicate that punishment of flight is itself not necessary for freezing to occur as the dominant reaction to threat.

The present study, therefore, suggests that an elicitation model, rather than a model involving the consequences of specific actions, may better predict the occurrence of flight and freezing reactions. In fact, even when the consequences (i.e., punishment or escape) of specific defensive reactions are followed by differential changes in the magnitude of further flight or freezing, it appears likely that this apparent relationship may in fact be mediated by alteration of the subject's familiarity with relevant features of the threat situation.

\section{REFERENCES}

Blanchard, R. J., Fukunaga, K. K., \& Blanchard, D. C. Environmental control of defensive reactions to $a$ cat. Bulletin of the Psychonomic Society, 1976, in press.

BlanchaRD, R. J., \& BlanchaRd, D. C. Defensive reactions in the albino rat. Learning and Motivation, 1971, 2, 351-362.

(Received for publication May 3, 1976.) 